\title{
Implementing quantum gates on oriented optical isomers
}

\author{
Ignacio R. Sola \\ Department of Chemistry, Princeton University, Princeton, New Jersey 08544 and Departamento \\ de Quimica Fisica I, Universidad Complutense, 28040 Madrid, Spain \\ Vladimir S. Malinovsky \\ Michigan Center for Theoretical Physics and FOCUS Center, Department of Physics, \\ University of Michigan, Ann Arbor, Michigan 48109 \\ Jesus Santamaría \\ Departamento de Quimica Fisica I, Universidad Complutense, 28040 Madrid, Spain
}

(Received 18 December 2003; accepted 22 March 2004)

\begin{abstract}
Optical enantiomers are proposed to encode molecular two-qubit information processing. Using sequences of pairs of nonresonant optimally polarized pulses, different schemes to implement quantum gates, and to prepare entangled states, are described. We discuss the role of the entanglement phase and the robustness of the pulse sequences which depend on the area theorem. Finally, possible scenarios to generalize the schemes to $n$-qubit systems are suggested. (C) 2004 American Institute of Physics. [DOI: 10.1063/1.1739403]
\end{abstract}

\section{INTRODUCTION}

Several quantum systems have been proposed as adequate, albeit often nonscalable, material support for implementing quantum gates and quantum circuits. ${ }^{1}$ A most natural system to encode the qubits is the spins of electrons and nuclei, since the dimension of the space is already binary for every half-spin particle. ${ }^{2}$ As molecular examples, there have been proposals to encode qubits in the electronic excitation of different chromophores ${ }^{3}$ or in the vibrational modes of molecules ${ }^{4}$ and polymer chains. ${ }^{5}$

In this work we propose the use of the handedness (related with parity) of an optical isomer as another quantum degree of freedom which can be easily used to encode a qubit. Molecules with high energy barriers for internal motion (like torsions) often present symmetry rules that correlate the transitions among eigenstates to the handedness or inversion with respect to that motion. The handedness also conforms a binary state space. Therefore, the combination of handedness and the hindered internal motion provides sufficient correlated degrees of freedom to implement simple conditional logic operations, i.e., quantum gates. The main restriction of this implementation is that the handedness cannot be used as a scalable resource. In the model that we consider in this work we concentrate on a four-level system and no claims are made towards using the system for quantum computation. Some possible extensions of the scheme to support a few-qubits are briefly discussed at the end of the paper.

Our main concern is to efficiently prepare nonseparable entangled states or, in general, coherent superpositions with precise control of the relative phase, adopting the language of Quantum Information science. ${ }^{1}$ The oriented optical isomer is an ideal system to transfer entanglement from the photon polarization of an optical source to the handedness of the molecule and vice versa. Therefore, the system may har- ness its main application in entanglement swapping ${ }^{6}$ or quantum information storage. ${ }^{1}$

The structure of the paper is the following: In Sec. II we introduce the properties of the system. In Sec. III we show several efficient ways of implementing quantum gates, and in Sec. IV we discuss possible extensions and we refer to the issue of scalability.

\section{PHYSICAL MODEL OF THE TWO-QUBIT SYSTEM}

\section{A. Required properties of the oriented optical isomer}

Let us consider, to be specific, a molecule such as $\mathrm{H}_{2} \mathrm{POSH}$. Steric effects hinder the torsion of the molecular $\mathrm{SH}$ fragment around the $\mathrm{P}-\mathrm{S}$ axis (see Fig. 1). In this example the electronic potential along the dihedral angle exhibits two wells separated by a moderate internal barrier, so that there are two ground conformations (cis and trans) that can only be interconverted by tunneling. Moreover both conformations are optical isomers (enantiomers) with different left/ right chirality. In the $\mathrm{H}_{2} \mathrm{POSH}$ example, both enantiomers interconvert by tunneling in $\sim 10 \mathrm{ps}^{7}$. The eigenstates of the molecule are grouped in pairs of even (positive) or odd (negative) parity $|n+\rangle,|n-\rangle$, where $n$ represents the number of nodes of the wave function in each well.

The electronic excited state $\left(S_{1}\right)$ of the molecule does not exhibit an internal barrier and the symmetry is such that the eigenstates $|m\rangle$ are also of even/odd parity for even/odd quanta excited on the torsional motion. The interesting property of the transient dipole moment is that depending on the direction it may be of even or odd parity. To be specific consider that for a chosen molecular axis-laser orientation, and for a particular chosen excited state, $\left\langle E\left|\mu_{\|}\right| n-\right\rangle$ $=0\left(\left\langle E\left|\mu_{\perp}\right| n+\right\rangle \neq 0\right) \quad$ and $\quad\left\langle E\left|\mu_{\perp}\right| n+\right\rangle=0\left(\left\langle E\left|\mu_{\|}\right| n-\right\rangle\right.$ $\neq 0)$. Hence, if the molecule cannot rotate (fixed orientation) a laser of perpendicular polarization $\mathcal{E}_{\perp}(t)$ can only induce transitions from the ground electronic potential with states of 


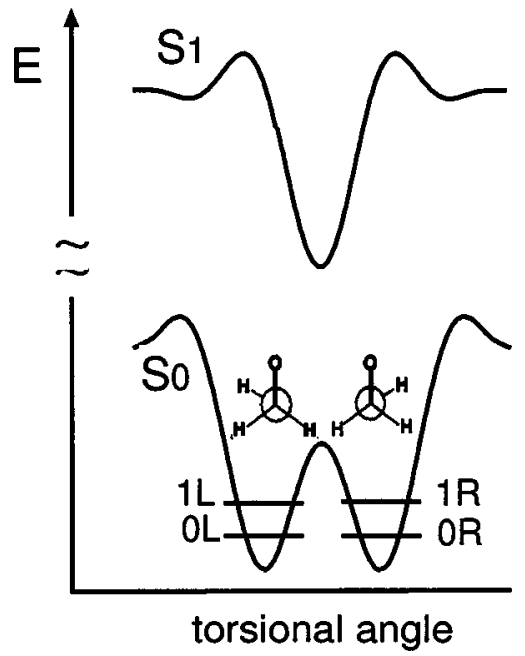

FIG. 1. Electronic potential curves of $\mathrm{H}_{2} \mathrm{POSH}$ showing the required features for the proposed two-qubit encoding of the system. Adapted from Ref. 7.

even parity, and conversely a laser of parallel polarization $\mathcal{E}_{\|}(t)$ can only induce transitions from the ground electronic potential with states of odd parity.

The previous symmetries are the required properties to implement in a particular molecule the schemes proposed in this work. These conditions are readily observed in several optical isomers. ${ }^{8,9}$ However, depending on the rate of tunneling, the molecular basis where the qubits are encoded must be chosen differently.

\section{B. Encoding the qubits on enantiomers with fast interconvertion}

For fast tunneling, the energy splitting between the pair of eigenstates $|n,+\rangle$ and $|n,-\rangle$, is typically large. By cooling, the molecule can be easily prepared in the state of lowest energy, $|0,+\rangle$. The eigenstates are then the most natural basis to encode the qubits. In a 2-qubits system, the encoding can be chosen as, e.g.,
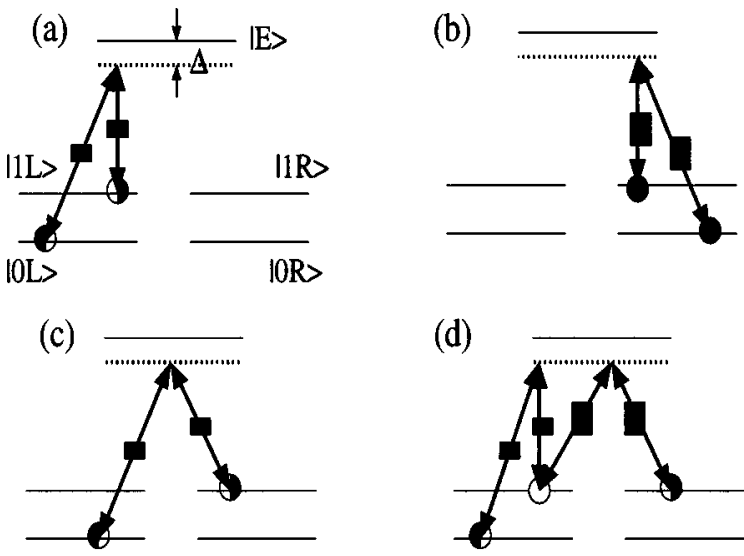

(d)
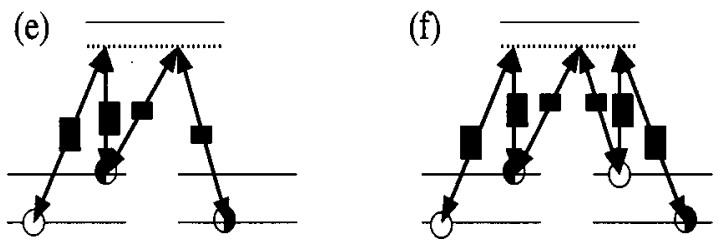

FIG. 2. Different schemes to implement one-qubit gates (the Hadamard gate, frame a), two-qubit gates (the c-NOT gate, frame b), and to prepare entangled states $\left.\left|\Phi^{+},\right| \Phi^{-}\right\rangle,\left|\Psi^{+}\right\rangle$, and $\left|\Psi^{-}\right\rangle$(frames c-f, respectively). The balls indicate the populations: empty (zero population), half-filled (50\% population) or filled (100\%); gray color indicates a change in the sign of the wave function. Every operation involves two pulses (two arrows). The required effective Rabi frequency $(\pi$ or $\pi / 2$ ) is indicated by the size of the square in the arrows.

$$
\begin{array}{ll}
|0+\rangle \leftrightarrow|00\rangle, & |0-\rangle \leftrightarrow|10\rangle, \\
|1+\rangle \leftrightarrow|01\rangle, & |1-\rangle \leftrightarrow|11\rangle,
\end{array}
$$

such that the first qubit represents the parity (even or odd) and the second qubit, the vibrational excitation. As shown in Fig. 2, the system uses an additional auxiliary state of the excited potential $|E\rangle$, to perform the operations. Selecting this state with a particular symmetry with respect to a chosen molecular orientation, as indicated in the previous section, the structure of the couplings can be described by the following Hamiltonian in the rotating wave approximation (RWA),

$$
\mathrm{H}=-\frac{1}{2}\left(\begin{array}{ccccc}
0 & 0 & 0 & 0 & \mathcal{E}_{0 \|}(t)\left\langle E\left|\mu_{\|}\right| 0+\right\rangle \\
0 & 0 & 0 & 0 & \mathcal{E}_{0 \perp}(t)\left\langle E\left|\mu_{\perp}\right| 0-\right\rangle \\
0 & 0 & 0 & 0 & \mathcal{E}_{1 \|}(t)\left\langle E\left|\mu_{\|}\right| 1+\right\rangle \\
0 & 0 & 0 & 0 & \mathcal{E}_{1 \perp}(t)\left\langle E\left|\mu_{\perp}\right| 0-\right\rangle \\
\mathcal{E}_{0 \|}(t)\left\langle E\left|\mu_{\|}\right| 0+\right\rangle & \mathcal{E}_{0 \perp}(t)\left\langle E\left|\mu_{\perp}\right| 0-\right\rangle & \mathcal{E}_{1 \|}(t)\left\langle E\left|\mu_{\|}\right| 1+\right\rangle & \mathcal{E}_{1 \perp}(t)\left\langle E\left|\mu_{\perp}\right| 0-\right\rangle & 2 \Delta
\end{array}\right) .
$$

We have chosen the pulse frequencies as: $\omega_{0 \|}=\omega_{E, 0+}-\Delta$, $\omega_{0 \perp}=\omega_{E, 0-}-\Delta, \omega_{1 \|}=\omega_{E, 1+}-\Delta, \omega_{1 \perp}=\omega_{E, 1-}-\Delta$ (where $\omega_{E, n \pm}$ is the frequency difference between $|E\rangle$ and $\left.|n \pm\rangle\right)$, such that all two-photon transitions between the qubit states are in resonance, but the auxiliary state can be off resonance by $\Delta$ with respect to every one-photon transition. The structure of this Hamiltonian allows very easy control of population transfer between the qubits and implementation of quan- 
tum gates. Different schemes will be developed in the next section. The drawback of the scheme is the need of four different lasers to operate $\left\{\mathcal{E}_{0 \|}(t), \mathcal{E}_{0 \perp}(t), \mathcal{E}_{1 \|}(t), \mathcal{E}_{1 \perp}(t)\right\}$.

\section{Encoding the qubits on enantiomers with slow interconvertion}

A more efficient implementation is possible using optical isomers with very high internal barriers. Then, the pure left/ right enantiomers are long live species. The pure enantiomers are superposition states of zero parity,

$$
\begin{aligned}
& |n L\rangle=(|n+\rangle+|n-\rangle) / \sqrt{2}, \\
& |n R\rangle=(|n+\rangle-|n-\rangle) / \sqrt{2},
\end{aligned}
$$

conforming a basis of localized states in each well. In fact, for very slow tunneling the localized basis is indeed more practical than the delocalized eigenstates, since optical isomers are typically found either in a given enantiomeric form, which is favored by chemical synthesis, or in a racemic (50:50) mixture, which is quickly obtained by decoherence from the delocalized energy states. In this situation we will assume that the initial state of the system is given by a localized state (e.g., $|0 L\rangle)$, which can be obtained by cooling the chemically preferred enantiomer, or by purifying the racemic mixture. ${ }^{7,10,11}$

Given that we are initially in $|0 L\rangle$, we encode a two qubit system as follows:

$$
\begin{array}{rlrl}
|0 L\rangle & \leftrightarrow|00\rangle, & |1 L\rangle \leftrightarrow|01\rangle, \\
|0 R\rangle \leftrightarrow|10\rangle, & |1 R\rangle \leftrightarrow|11\rangle,
\end{array}
$$

so that the first qubit represents the handedness of the optical isomer, while the second qubit represents the vibrational excitation. Besides the four level (two-qubit) system, we use the state $|E\rangle$ in the excited electronic potential as a bus state where the information is not encoded.

In this system there are only two active frequencies or pulses. By controlling the polarization of the fields we need to prepare the specific pulses that will selectively drive the four transitions between the localized states (not the eigenstates) and the $|E\rangle$ state. ${ }^{12}$ Since the basis is rotated, the optimal polarizations must be rotated with respect to the perpendicular and parallel orientations. For instance, $\mathcal{E}(t)$ $=\mathcal{E}_{0 \perp}(t) \mathbf{e}_{\perp}$ is active in both transitions from $|0 L\rangle$ and $|0 R\rangle$ to $|E\rangle$. A linear combination $\mathcal{E}_{0 L}(t)=\mathcal{E}_{0 \perp}(t) \mathbf{e}_{\perp}+\mathcal{E}_{0 \|}(t) \mathbf{e}_{\|}$is needed such that $\left\langle E\left|\mathcal{E}_{0 L}(t) \cdot \boldsymbol{\mu}\right| 0 R\right\rangle=0$ but $\langle E| \mathcal{E}_{0 L}(t)$ $\cdot \boldsymbol{\mu}|0 L\rangle \neq 0$. The first condition implies that

$$
\begin{aligned}
& \mathcal{E}_{0 \perp}(t)\left\langle E\left|\mu_{\perp}\right| 0 R\right\rangle+\mathcal{E}_{0 \|}(t)\left\langle E\left|\mu_{\|}\right| 0 R\right\rangle \\
& \quad=-\mathcal{E}_{0 \perp}(t)\left\langle E\left|\mu_{\perp}\right| 0-\right\rangle+\mathcal{E}_{0 \|}(t)\left\langle E\left|\mu_{\|}\right| 0+\right\rangle=0,
\end{aligned}
$$

which can be easily satisfied by choosing the field

$$
\mathcal{E}_{0 L}(t)=\mathcal{E}_{0} \mathcal{S}_{0}(t) \mathbf{e}_{0 L}
$$

with the specific linear polarization

$$
\begin{aligned}
\mathbf{e}_{0 L}= & \frac{\left\langle E\left|\mu_{\|}\right| 0+\right\rangle}{\sqrt{\left|\left\langle E\left|\mu_{\perp}\right| 0-\right\rangle\right|^{2}+\left|\left\langle E\left|\mu_{\|}\right| 0+\right\rangle\right|^{2}}} \mathbf{e}_{\perp} \\
& +\frac{\left\langle E\left|\mu_{\perp}\right| 0-\right\rangle}{\sqrt{\left|\left\langle E\left|\mu_{\perp}\right| 0-\right\rangle\right|^{2}+\left|\left\langle E\left|\mu_{\|}\right| 0+\right\rangle\right|^{2}}} \mathbf{e}_{\|},
\end{aligned}
$$

where $\mathcal{E}_{0}$ is the peak amplitude and $\mathcal{S}_{0}(t)$ is the envelope function. With this choice of polarization we observe that $\left\langle E\left|\mathbf{e}_{0 L} \cdot \boldsymbol{\mu}\right| 0 L\right\rangle \neq 0$. Using the same procedure we obtain

$$
\mathcal{E}_{0 R}(t)=\mathcal{E}_{0} \mathcal{S}_{0}(t) \mathbf{e}_{0 R}
$$

with

$$
\begin{aligned}
\mathbf{e}_{0 R}= & -\frac{\left\langle E\left|\mu_{\|}\right| 0+\right\rangle}{\sqrt{\left|\left\langle E\left|\mu_{\perp}\right| 0-\right\rangle\right|^{2}+\left|\left\langle E\left|\mu_{\|}\right| 0+\right\rangle\right|^{2}}} \mathbf{e}_{\perp} \\
& +\frac{\left\langle E\left|\mu_{\perp}\right| 0-\right\rangle}{\sqrt{\left|\left\langle E\left|\mu_{\perp}\right| 0-\right\rangle\right|^{2}+\left|\left\langle E\left|\mu_{\|}\right| 0+\right\rangle\right|^{2}}} \mathbf{e}_{\|} .
\end{aligned}
$$

Usually the dipole moments in the transitions from the odd/even parity eigenstates are approximately equal, $\left\langle E\left|\mu_{\|}\right| 0+\right\rangle \approx\left\langle E\left|\mu_{\perp}\right| 0-\right\rangle$. Then, the optimal polarizations for the pulses are perpendicular $\mathbf{e}_{0 R} \cdot \mathbf{e}_{0 L} \approx 0$. The expressions for $\mathcal{E}_{1 L}(t)=\mathcal{E}_{1} \mathcal{S}_{1}(t) \mathbf{e}_{1 L}$ and $\mathcal{E}_{1 R}(t)=\mathcal{E}_{1} \mathcal{S}_{1}(t) \mathbf{e}_{1 R}$ are obtained substituting $|0+\rangle$ and $|0-\rangle$ by $|1+\rangle$ and $|1-\rangle$ in Eqs. (5) and (6), respectively.

With the choice of the optimal polarizations we obtain the four lasers $\left\{\mathcal{E}_{0 L}(t), \mathcal{E}_{0 R}(t), \mathcal{E}_{1 L}(t), \mathcal{E}_{1 R}(t)\right\}$ that operate in the different transitions. However, the physical resources are only two laser fields. It is important to realize that the only difference between $\mathcal{E}_{n L}(t)$ and $\mathcal{E}_{n R}(t)$ is the direction of polarization. When $\mathbf{e}_{n L}$ and $\mathbf{e}_{n R}$ are not orthogonal, the pulses cannot act at the same time, since its sum will be another pulse of different polarization. Having this restriction in mind, the same Hamiltonian in Eq. (2) applies, substituting the perpendicular or parallel polarizations by the proper optimized polarizations.

\section{SCHEMES FOR IMPLEMENTING QUANTUM GATES}

Once the Hamiltonian has been defined by the proper encoding of qubits and the preparation of pulses, in this section we propose different schemes or sequences of pulses that can be used to implement one and two-qubit gates, and to prepare maximally entangled Bell states. We concentrate on the schemes based on molecules with very slow tunneling, where the basis are pure enantiomers or localized superposition states. The same schemes can be applied to molecules with fast tunneling, changing the encoding to the delocalized basis and the optimal field polarizations to the perpendicular and parallel components. We refer here to optical schemes based on off resonant Raman with fully overlapping pulses, in which case we use the same envelope function for all pulses $\mathcal{S}_{0}(t)=\mathcal{S}_{1}(t) \equiv \mathcal{S}(t)$. This is the simplest scheme that allows the required control in our physical system. Other schemes using more complex pulse sequences are possible. They are briefly outlined in the final discussion. 
As a counterexample of a nonworking scheme, it is not possible to use resonant fully overlapping pulses to prepare 50:50 superposition states of the qubits. ${ }^{13}$

\section{A. Implementing one-qubit gates: The Hadamard gate as a $\pi / 2$ transformation}

As an example of a one-qubit gate we show the necessary steps to prepare the Hadamard gate, for instance on the first qubit. The Hadamard gate $(\mathcal{H})$ operates such that

$$
\begin{aligned}
& \mathcal{H}|0 L\rangle=(|0 L\rangle+|1 L\rangle) / \sqrt{2}, \\
& \mathcal{H}|1 L\rangle=(|0 L\rangle-|1 L\rangle) / \sqrt{2} .
\end{aligned}
$$

Using two fully overlapping nonresonant pulses, $\mathcal{E}_{0 L}(t)$ and $\mathcal{E}_{1 L}(t)$, with a sufficiently large detuning to the excited state $|E\rangle$, the right localized states are decoupled and the excited state is a virtual state [see for reference the Hamiltonian (2)]. The amplitude of probability in $|E\rangle$ can be considered small and constant. We also choose the peak amplitudes such that

$$
\mathcal{E}_{0} \mathcal{S}(t) \boldsymbol{\mu} \cdot \mathbf{e}_{0 L}=\mathcal{E}_{1} \mathcal{S}(t) \boldsymbol{\mu} \cdot \mathbf{e}_{0 R} \equiv \Omega_{0}(t) .
$$

By adiabatic elimination of the excited state $|E\rangle$ we obtain, in the RWA, the effective Hamiltonian that acts only in the first qubit

$$
\mathrm{H}_{1 L, 0 L}=\frac{\Omega_{0}^{2}(t)}{4 \Delta}\left(\begin{array}{ll}
1 & 1 \\
1 & 1
\end{array}\right)
$$

The Schrödinger equation can be solved analytically, $|\Psi(t)\rangle=\bigcup(t, 0)|\Psi(0)\rangle$,

$$
\bigcup_{1 L, 0 L}(t, 0)=e^{-i \mathcal{A} / 2}\left(\begin{array}{cc}
\cos (\mathcal{A} / 2) & -i \sin (\mathcal{A} / 2) \\
-i \sin (\mathcal{A} / 2) & \cos (\mathcal{A} / 2)
\end{array}\right),
$$

where for a pulse of duration $T$, the pulse area is defined by

$$
\mathcal{A}=\int_{0}^{T} d t \Omega_{0}^{2}(t) / 2 \Delta \text {. }
$$

By choosing $\mathcal{A}=\pi / 2$ we prepare 50:50 superposition states. We call this transformation $\bigcup_{1 L, 0 L}^{\pi / 2}$, which gives (besides an unimportant overall phase factor):

$$
\begin{aligned}
& \bigcup_{1 L, 0 L}^{\pi / 2}|0 L\rangle=(|0 L\rangle-i|1 L\rangle) / \sqrt{2}, \\
& \bigcup_{1 L, 0 L}^{\pi / 2}|1 L\rangle=(|0 L\rangle+i|1 L\rangle) / \sqrt{2} .
\end{aligned}
$$

$\mathrm{U}_{1 L, 0 L}^{\pi / 2}$ works exactly as the Hadamard gate. The procedure is schematically shown in Fig. 2(a). The mismatch of the relative phase can be incorporated in the definition of the basis, $|1 L\rangle \rightarrow e^{-i \pi / 2}|1 L\rangle$, so that there are no observable (i.e., physical) differences between $\bigcup_{1 L, 0 L}^{\pi / 2}$ and $\mathrm{H}$.

\section{B. Implementing two-qubits gates: The c-NOT gate as a $\pi$ transformation}

Choosing the molecular handedness as the control qubit (with the left enantiomer as the 0 bit) the c-NOT gate is simply a flip in the population on the right-hand enantiomer and not in the left-hand enantiomer,

$$
\begin{array}{ll}
\mathcal{N} 10 L\rangle=|0 L\rangle, & \mathcal{M} 0 R\rangle=|1 R\rangle, \\
\mathcal{N} 1 L\rangle=|1 L\rangle, & \mathcal{M} 1 R\rangle=|0 R\rangle .
\end{array}
$$

By using nonresonant fully overlapping $\mathcal{E}_{0 R}(t)$ and $\mathcal{E}_{1 R}(t)$ pulses, the first (left-hand) qubit is not coupled, and the lasers will only interact with the second (right-hand qubit). The Hamiltonian of the interaction is $\mathrm{H}_{1 R, 0 R}$ [Eq. (9)] and the solution of the dynamics is $\bigcup_{1 R, 0 R}(t, 0)[\mathrm{Eq}$. (10)] with the " $R$ " label instead of the " $L$ " label. Full population inversion is obtained by choosing $\mathcal{A}=\pi$ (or odd multiples of $\pi$ ). We call this solution $\mathrm{U}_{1 R, 0 R}^{\pi}$,

$$
\begin{aligned}
& \bigcup_{1 R, 0 R}^{\pi}|0 R\rangle=-|1 R\rangle, \\
& \bigcup_{1 R, 0 R}^{\pi}|1 R\rangle=-|0 R\rangle .
\end{aligned}
$$

Notice that the overall phase is unimportant and therefore $\mathrm{U}_{1 R, 0 R}^{\pi}$ is exactly the c-NOT gate. However, we keep the phase factor since it can be relevant in sequential gate operations that act on part of the system, as shown in the next subsection.

Obviously, the definition of the c-NOT gate depends on the nature of the encoding, which is arbitrary. If, for instance, the control qubit is changed to the first qubit, then the same scheme can be applied by rotating the polarization of the pulses, that is, by using $\mathcal{E}_{0 L}(t)=\mathcal{E}_{0} \mathcal{S}(t) \mathbf{e}_{0 L}$ and $\mathcal{E}_{1 L}(t)$ $=\mathcal{E}_{1} \mathcal{S}(t) \mathbf{e}_{1 L}$, in which case the c-NOT gate would be equal to $\mathrm{U}_{0 L, 1 L}^{\pi}$.

\section{Preparing entangled states: Phase control of superpositions by multiple-steps}

In order to prepare the entangled state

$$
\left|\Phi^{ \pm}\right\rangle=(|00\rangle \pm|11\rangle) / \sqrt{2}=(|0 L\rangle \pm|1 R\rangle) / \sqrt{2},
$$

from the initial state $|0 L\rangle$, we need to use the pulses $\mathcal{E}_{0 L}(t)$ and $\mathcal{E}_{1 R}(t)$. In this case, the other states of the system are decoupled, and the same Hamiltonian $\mathrm{H}_{1 R, 0 L}$ [Eq. (9)] represents the interaction between the required states. $\mathrm{U}_{1 R, 0 L}(t, 0)$ [Eq. (10)] gives again the system dynamics. For pulse areas $\mathcal{A}=\pi / 2, \mathrm{U}_{1 R, 0 L}^{\pi / 2}$ is constructed, which acts exactly in the same way as $\bigcup_{1 L, 0 L}^{\pi / 2}$ [see Eq. (12)] when $|1 L\rangle$ is substituted by $|1 R\rangle$. By redefinition of the basis phase, $\mathrm{U}_{1 R, 0 L}^{\pi / 2}$ can be used to prepare one of the entangled states, but not both of them, since the phase, although arbitrary, must be fixed. To be specific, we consider hereafter $e^{-i \pi / 2}|0 R\rangle \rightarrow|0 R\rangle$, and $e^{-i \pi / 2}|1 R\rangle \rightarrow|1 R\rangle$. Then

$$
\mathrm{U}_{1 R, 0 L}^{\pi / 2}|0 L\rangle=(|0 L\rangle-i|1 R\rangle) / \sqrt{2} \equiv\left|\Phi^{+}\right\rangle .
$$

We need to devise and alternative strategy to change the relative phase of the superposition and prepare the orthogonal Bell state $\left|\Phi^{-}\right\rangle$.

A simple way of controlling the phase is by performing sequential operations. The $\bigcup_{i, j}^{\pi}$ transformation adds a $\pi$ phase to the wave function at the same time that the population is transferred. Therefore, there should be as many population flips as phase shifts are required. In order to prepare $\left|\Phi^{-}\right\rangle$from $|0 L\rangle$ the simplest 2-pulse sequence could be

$$
\left|\Phi^{-}\right\rangle=\bigcup_{1 R, a}^{\pi} \bigcup_{a, 0 L}^{\pi / 2}|0 L\rangle,
$$

where $|a\rangle$ can be any other qubit state, for instance $|1 L\rangle$. The first transformation $\bigcup_{1 L, 0 L}^{\pi / 2}$ creates a 50:50 superposition of $|0 L\rangle$ and an intermediate unwanted state $(|1 L\rangle)$; the second transformation $\bigcup_{1 R, 1 L}^{\pi}$ moves all the population from the 
unwanted state to the desired final state. Since one $\pi$ transformation has been added to the former scheme, the final superposition changes a $\pi$ phase. Notice that for the same reasons, it is not possible to prepare superposition states of the form $|a\rangle \pm|b\rangle$ without phase redefinition. This fact is related with the impossibility of changing the handedness of a state by interactions that do not distinguish the handedness. ${ }^{14}$

The same ideas can be applied to prepare entangled states of the form

$$
\left|\Psi^{ \pm}\right\rangle=(|01\rangle \pm|10\rangle) / \sqrt{2}=(|1 L\rangle \mp i|0 R\rangle) / \sqrt{2},
$$

where we have already incorporated the proper phase redefinition. In this case, since the initial state is not part of the desired final superposition, we need to move all the population from $|0 L\rangle$. We can, for instance, first operate on the first qubit by flipping the population to one of the final states, e.g., $|1 L\rangle$, and then partially transfer the population from this state to the other state of the target superposition, e.g., $|0 R\rangle$,

$$
\left|\Psi^{+}\right\rangle=\bigcup_{0 R, 1 L}^{\pi / 2} \bigcup_{1 L, 0 L}^{\pi}|0 L\rangle .
$$

This is a $\pi$ followed by $\pi / 2$ sequence. An alternative scheme is

$$
\left|\Psi^{+}\right\rangle=\bigcup_{0 R, 0 L}^{\pi} \bigcup_{1 L, 0 L}^{\pi / 2}|0 L\rangle,
$$

which involves a $\pi / 2$ followed by a $\pi$ transformation, as in the preparation of $\left|\Phi^{-}\right\rangle$.

We need an additional $\pi$ transformation (a 3 pulse sequence) to obtain the $\left|\Psi^{-}\right\rangle$entangled state. A particular realization is, for instance,

$$
\left|\Psi^{-}\right\rangle=\mathrm{U}_{0 R, a}^{\pi / 2} \mathrm{\cup}_{a, 1 L}^{\pi / 2} \mathrm{U}_{1 L, 0 L}^{\pi}|0 L\rangle,
$$

where $|a\rangle$ is any intermediate unwanted state, e.g., $|1 R\rangle$. Possible realizations of the entangled states are schematically shown in Figs. 2(c) -2 (f).

The procedure presented above is not constrained to the particular arbitrary encoding that is chosen. The language of transformations is universal. If the subindexes in $\bigcup_{i, j}^{\pi}$ and $\bigcup_{k, l}^{\pi / 2}$ label directly the qubits $\{|00\rangle,|01\rangle,|10\rangle,|11\rangle\}$, instead of the localized or delocalized Hamiltonian basis states, then the transformations represent general realizations of the gates. The implementation of a particular transformation requires the choice of the two nonresonant (optimally polarized) fully overlapping pulses, which obviously depends on the choice of encoding.

\section{Numerical results}

We have solved the Schrödinger equation (2) assuming, without loss of generality, that all the Franck-Condon factors are equal to 1 . Then $\mathbf{e}_{0 L}=\mathbf{e}_{1 L}=\left(\mathbf{e}_{\|}+\mathbf{e}_{\perp}\right) / \sqrt{2}$ and $\mathbf{e}_{0 R}$ $=\mathbf{e}_{1 R}=\left(-\mathbf{e}_{\|}+\mathbf{e}_{\perp}\right) / \sqrt{2}$. Using units scaled with the time width of the pulses $(\tau=1)$, we fix $\Delta=8 \pi^{3 / 2} \approx 44.5$ and we implement the schemes based on fully overlapping nonresonant pair of pulses, as explained previously. For Gaussian shaped pulses $\left(\int_{-\infty}^{\infty} d t e^{-t^{2}}=\sqrt{2}\right)$

$$
\mathcal{A}=\sqrt{\pi} \Omega_{0}^{2} / 2 \Delta .
$$
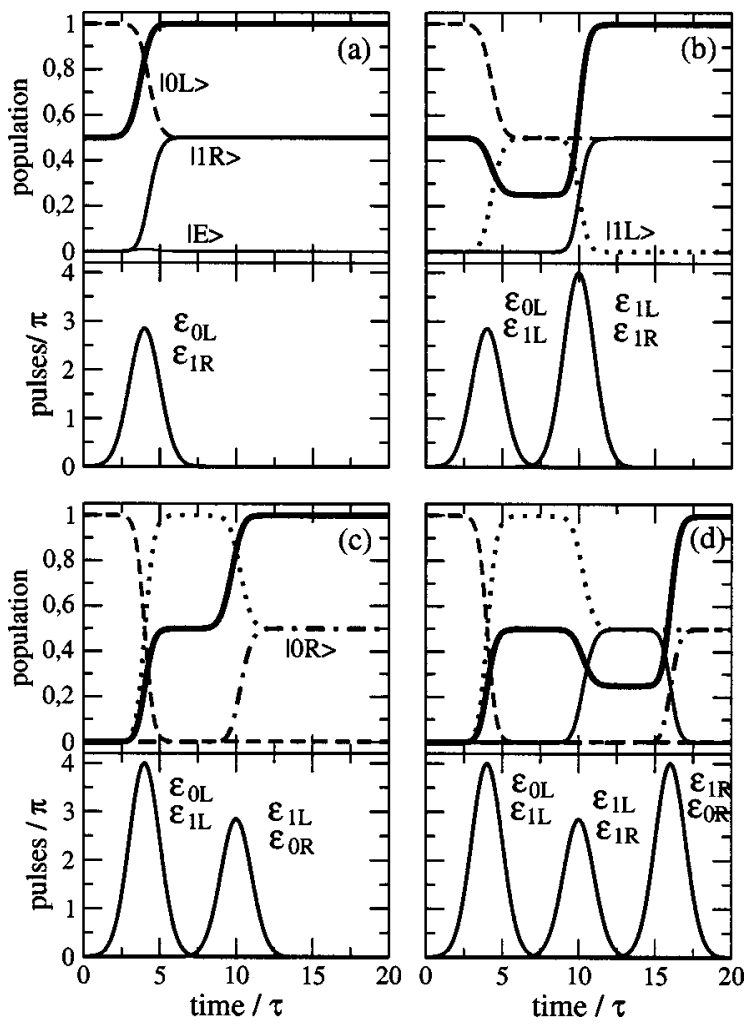

FIG. 3. Dynamics for the controlled preparation of entangled states $\left|\Phi^{+}\right\rangle$, $\left|\Phi^{-}\right\rangle,\left|\Psi^{+}\right\rangle$, and $\left|\Psi^{-}\right\rangle$(frames a-d, respectively). We show the population histories in the qubit basis $(|0 L\rangle$, dashed line; $|0 R\rangle$, dotted-dashed line; $|1 L\rangle$, dotted line; $|1 R\rangle$, solid line) and in the prepared entangled state (thick gray line) as well as the shape of every pair of laser pulses.

Therefore, for a $\pi$ pulse transformation the area of every pulse in the pair must be $\Omega_{0}^{\pi}=4 \pi$, and for a $\pi / 2$ transformation $\Omega_{0}^{\pi / 2}=\sqrt{8} \pi$.

Using the theoretically predicted values, Fig. 3 shows the results for preparing the different entangled states discussed in the previous section. The yield of the efficiency in each case was higher than 0.995 , and almost no population was transferred to the excited state.

From a physical point of view, if the molecular density of states in the enantiomer is around one per wave number, $\rho \sim 1 \mathrm{~cm}$, then the transformations could be performed using pulses with time widths $\tau \sim 100$ ps and peak intensities of the order of $10^{5} \mathrm{~W} / \mathrm{cm}^{2}$ for realistic Franck-Condon factors of the order of 0.1 .

To prepare the different $\pi$ and half $\pi$ pulses we have fixed the detuning and time width, optimizing the field amplitudes. Depending on the experimental needs the pulses could be prepared optimizing only the time widths or the carrier frequencies (detunings).

\section{DISCUSSION}

The parity or handedness and the torsional motion of an optical isomer configure a double-well potential. In some cases, the symmetry of the dipole moments and the excited state in the oriented isomer allow for simple implementations of quantum gates. Depending on the tunneling rates, we have proposed two different encodings for the qubits. The same schemes can be applied for the preparation of the gates and entangled states in both cases. However, some restrictions 
apply to the phases of the entangled states that can be prepared. The general strategy is based on sequences of pairs of nonresonant fully overlapping pulses with specific polarizations and $\pi$ or half $\pi$ pulse areas, that drive selective excitations. This constitutes the simplest possible strategy, particularly for molecules with slow tunneling rates, where only two pulses (with the same shape) are needed.

There are many other possible implementations of the gates. Recently we have proposed the use of phase-control of partially time-delayed pulse sequences, both in and off resonance with the excited state, to prepare entanglement in four level systems. ${ }^{15}$ The area of the pulses must be relatively large, but the advantage of these schemes is a greater insensitivity to the pulse area. The same schemes can be applied to the optical isomer. Moreover, the symmetry of the couplings in the oriented optical isomer allows to use polarization control instead of the phase control of the pulses.

The advantage of using off-resonant sequences is that the population remains all the time in the qubits space; it never reaches the excited electronic potential, avoiding possible dissociation or fluorescence processes. The disadvantage is the need of using stronger pulses since the Rabi frequency depends on the square of the pulse amplitude. For instance, in our numerical results, the amplitude of the optimal pulses for the $\pi$ pulse transformation is $4 \pi$, instead of $\pi$. In any case, it can be proven that it is not possible to prepare the required superposition states by using resonant fully overlapping pulses. ${ }^{13}$ Therefore all resonant schemes must use time delayed pulse sequences, leading to substantial population in the excited states, or more complicated pulse sequences, as mentioned above.

The main problems for the experimental implementation of the proposal in this work are two: the arrangement of the oriented isomers and the preparation of the initial state. In molecules with slow tunneling the initial sample must be a single enantiomer in the ground state. As in NMR-based quantum computation, ${ }^{2}$ the sensitivity of the information processing will depend on the different initial populations on the left and right enantiomers. However, there have been quite promising proposals to purify initial racemic mixtures (50:50 mixture of left/right enantiomers) using optical methods. ${ }^{7,10-12}$ Some of these strategies hint the possibility of implementing schemes that use initially polarized isomers, instead of oriented samples. ${ }^{8,11}$

We have alluded to the difficulty of extending these schemes to $N$-qubit systems. It is in principle possible to implement more qubits in the vibrational levels of the double well. For instance, by using 4 states in each enantiomer it is possible to design three qubit operations. The problem is that the number of pulses needed to control the operations in each enantiomer will grow exponentially. This scalability problem prevents the encoding of many qubits per enantiomer. Another possibility is the use of diastereomers, that is, molecules with several enantiomeric centers, for instance, proteins. These molecules could work as strings of double well potentials. If the symmetry conditions are favorable, it could be possible to find adequate pulse polarizations to encode two (or a few) qubits and perform quantum logic operations by implementing the previously proposed schemes (or simple extensions) in every double well. The difficulty in the scalability problem is the entangling (i.e., connection) of the different units, a prerequisite for any complex quantum computation. More studies are needed to assess the viability of $\mathrm{N}$-qubit entanglement and quantum information processing in large stringlike molecules. However, we believe that the present contribution presents a new family of molecular candidates at least for implementing simple two-qubit operations and entanglement, and shows new ways of looking at and using optical isomers.

\section{ACKNOWLEDGMENTS}

This work was partially supported by the National Science Foundation through a grant for the Institute of Theoretical Atomic and Molecular Physics at Harvard University and the Smithsonian Astrophysical Observatory, and by the Dirección General de Investigación Científica y Técnica of Spain under Project No. BQU2002-00173. I.R.S. thanks L. González for introducing him to the Quantum Chemistry of enantiomers and for providing Fig. 1.

${ }^{1}$ D. Bouwmeester, A. Ekert, and A. Zeilinger, The Physics of Quantum Information (Springer Verlag, Berlin, 2000).

${ }^{2}$ M. A. Nielsen and I. L. Chuang, Quantum Computation and Quantum Information (Cambridge University Press, Cambridge, 2000).

${ }^{3}$ J. P. Palao and R. Kosloff, Phys. Rev. Lett. 89, 188301 (2002); E. A. Shapiro, M. Spanner, and M. Yu. Ivanov, ibid. 91, 237901 (2003).

${ }^{4}$ C. M. Tesch and R. de Vivie-Riedle, Phys. Rev. Lett. 89, 157901 (2002).

${ }^{5}$ S. Lloyd, Science 261, 1569 (1993).

${ }^{6}$ S. Bose, V. Vedral, and P. L. Knight, Phys. Rev. A 57, 822 (1998); J.-W. Pan, D. Bouwmeester, H. Weinfurter, and A. Zeilinger, Phys. Rev. Lett. 80, 3891 (1998).

${ }^{7}$ Y. Fujimura, L. González, K. Hoki, D. Kroner, J. Manz, and Y. Ohtsuki, Angew. Chem., Int. Ed. Engl. 39, 4586 (2000); L. González, D. Kröner, and I. R. Sola, J. Chem. Phys. 115, 2519 (2001).

${ }^{8}$ Y. Fujimura, L. González, K. Hoki, J. Manz, and Y. Ohtsuki, Chem. Phys. Lett. 306, 1 (1999); 310, 578(E) (1999).

${ }^{9}$ E. Deretey, M. Shapiro, and P. Brumer, J. Phys. Chem. A 105, 9509 (2001); M. Shapiro and P. Brumer, Rep. Prog. Phys. 66, 859 (2003).

${ }^{10}$ A. Salam and W. J. Meath, J. Chem. Phys. 106, 7865 (1997); K. Hoki, L. González, and Y. Fujimura, ibid. 116, 2433 (2002); Y. Ohta, K. Hoki, and Y. Fujimura, ibid. 116, 7509 (2002).

${ }^{11}$ M. Shapiro, E. Frishman, and P. Brumer, Phys. Rev. Lett. 84, 1669 (2000); E. Frishman, M. Shapiro, D. Gerbasi, and P. Brumer, J. Chem. Phys. 119, 7237 (2003).

${ }^{12}$ K. Hoki, L. González, and Y. Fujimura, J. Chem. Phys. 116, 8799 (2002); D. Kröner and L. González, Chem. Phys. 298, 55 (2004).

${ }^{13}$ For fully overlapping resonant pulses $(\Delta=0)$ connecting qubit states $|i\rangle$ and $|f\rangle$ with the auxiliary state $|E\rangle$ by the proper pulse polarizations with pulse area matching conditions: $\boldsymbol{\mu} \mathcal{E}_{i}(t)=\boldsymbol{\mu} \mathcal{E}_{j}(t)=\Omega_{0}(t)$, the Hamiltonian of the interaction among the coupled states in the RWA is

$$
\mathrm{H}_{i, f, E}=-\left[\Omega_{0}(t) / 2\right]\left(\begin{array}{lll}
0 & 0 & 1 \\
0 & 0 & 1 \\
1 & 1 & 0
\end{array}\right) .
$$

The Schrödinger equation for this Hamiltonian has analytical solution. If the initial state is in $|i\rangle$, the final wave function will be $|\psi(t)\rangle=\frac{1}{2}(1$ $+\cos \mathcal{A})|i\rangle-\frac{1}{2}(1-\cos \mathcal{A})|f\rangle+(i / \sqrt{2}) \sin \mathcal{A}|E\rangle$, where $\mathcal{A}=\int \Omega(t) d t$ is the pulse area. Population inversion from $|i\rangle$ to $|f\rangle$ is possible using $\pi$ pulses. However, it is not possible to create 50:50 superposition states of $|i\rangle$ and $|f\rangle$ and at the same time avoid population in $|E\rangle$, that is, every superposition or entangled state prepared by resonant fully overlapping pulses would be contaminated by some population in the excited electronic state. In fact, for half $\pi$ pulses $|\psi(t)\rangle=(|i\rangle-|f\rangle) / \sqrt{2}+i|E\rangle / \sqrt{2}$ so that $50 \%$ of the population is actually in $|E\rangle$.

${ }^{14}$ C. S. Maierle and R. A. Harris, J. Chem. Phys. 109, 3713 (1998).

${ }^{15}$ V. S. Malinovsky and I. R. Sola (unpublished). 